\title{
Magnetic Measurements for a MICE Spectrometer Solenoid
}

\author{
M. Buehler, A. Bross, C. Hess, D. Orris, R. Pilipenko, \\ R. Preece, C. Sylvester, M. Tartaglia, J. Tompkins, S. Virostek
}

\begin{abstract}
The goal of the Muon Ionization Cooling Experiment (MICE) is to demonstrate muon cooling for a future muon collider. In order to quantify this cooling effect with high precision, scintillating fiber trackers in a uniform 4 Tesla field are required. The MICE spectrometer solenoids were designed to meet these requirements. Based on superconducting NiobiumTitanium (Nb-Ti) each of the two MICE spectrometers solenoids consists of five separate coils contained in a vacuum vessel of 2.7 $\mathrm{m}$ length and $1.4 \mathrm{~m}$ diameter. In this paper we report on results from first measurements to verify initial magnet performance at the manufacturer site using a portable Hall-probe-based measurement system. A comparison with theoretical expectations based on OPERA simulations will be discussed and design aspects of the measurement system will be presented.
\end{abstract}

Index Terms-Muon colliders, Solenoids, Superconducting coils

\section{INTRODUCTION}

$\mathrm{T}$ HE CONCEPT of a Muon Collider, while technically very challenging, provides several advantages over existing proposals for future accelerator technologies. Since muons are fundamental particles, the energy of the interaction is the full energy of the colliding particles enabling precision measurements. Bremsstrahlung from muons is reduced by a factor of $\left(\mathrm{m}_{\mathrm{e}} / \mathrm{m}_{\mu}\right)^{4} \sim 6 \times 10^{-10}$ as compared to electrons. This results in much reduced power consumption and a large overall reduction in size for a Muon Collider facility. Furthermore, neutrinos from muon decays can be used as a source for a Neutrino Factory, which would provide an upgrade path for a strong Intensity Frontier program [1].

The short lifetime of the muon particles $(\sim 2.2 \mu \mathrm{s})$ results in several technical challenges that need to be addressed in order to be able to generate high intensity muon beams. Particularly, reducing the transverse emittance of a beam of muons in order to produce very bright, focused beams that can be accelerated to high energies needs to be demonstrated. The Muon Ionization Cooling Experiment (MICE) is an R\&D project that aims to demonstrate the "cooling" of muon beams.

Manuscript received October 9, 2012. This work was supported in part by Fermi Research Alliance, LLC, under contract No. DE-AC02-07CH11359 with the U.S. Department of Energy.

M. Buehler, A. Bross, C. Hess, D. Orris, R. Pilipenko, C. Sylvester, M. Tartaglia, and J. Tompkins are with the Fermi National Accelerator Laboratory, Batavia, IL 80510 USA (corresponding author phone: 630-8404710; fax 630-840-8079; e-mail: buehler@fnal.gov.).

R. Preece is with the Rutherford Appleton Laboratory, Harwell Oxford, UK.

S. Virostek is with the Lawrence Berkeley National Laboratory, Berkeley, CA, USA.
Fig. 1 illustrates the setup for the MICE cooling channel which is to be installed at Rutherford Appleton Laboratory (RAL) in England. As the muon beam enters the cooling channel, the initial emittance will be measured within the first of two spectrometer solenoids. Liquid Hydrogen absorbers reduce both longitudinal and transverse momentum components of the muon beam, while acceleration in radio frequency (RF) cavities restores the longitudinal component, thus providing a net "cooling" effect. A second spectrometer solenoid is used to verify the cooling of the muon beam.

\section{MICE SPECTROMETER SOLENOIDS}

Each MICE spectrometer solenoid consists of five superconducting $\mathrm{Nb}-\mathrm{Ti}$ coils wound on a common aluminum mandrel. Two Matching Coils (M1, M2) match the muon beam in the spectrometer solenoids with the beam in the adjacent absorber modules. Two End Coils (E1, E2) positioned around a Center Coil (C) will generate a $4 \mathrm{~T}$ uniform field over a 1-m-long and 30-cm-diameter volume (Table I) [2].

The cold mass is contained within a cryostat which consists of a liquid helium vessel, a thermal shield, cold mass supports, and a stainless steel vacuum vessel $(2.7 \mathrm{~m}$ in length, and $1.4 \mathrm{~m}$ in diameter). An array of cryo coolers is used to cool the cold mass and thermal shield [3].

An FPGA-based quench detection and continuous logging system were used for testing [4].

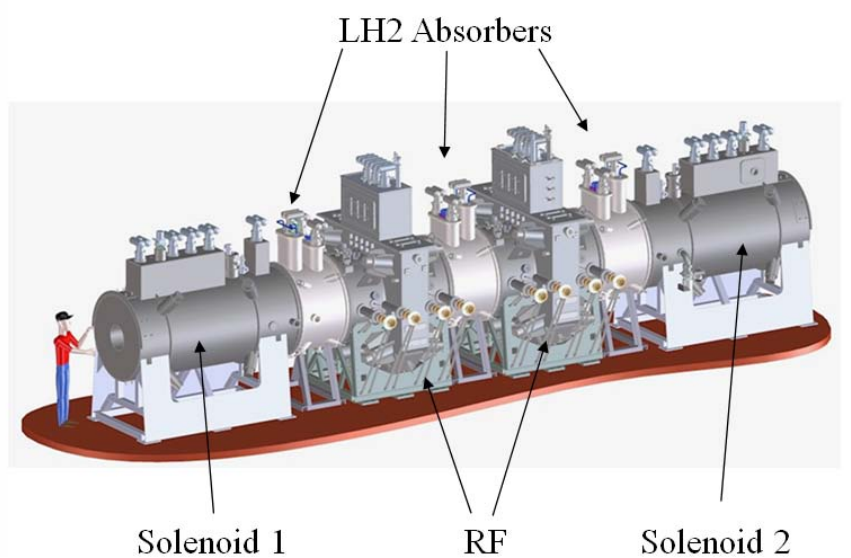

Fig. 1. MICE cooling channel depicting spectrometer solenoids, liquid hydrogen (LH2) absorbers, and RF cavities. 
TABLE I

MICE SPECTROMETER SOLENOID AS-BUILT PARAMETERS

\begin{tabular}{|c|c|c|c|c|c|}
\hline & Match 1 & Match 2 & End 1 & Center & End 2 \\
\hline Inner Coil Radius (mm) & 258 & 258 & 258 & 258 & 258 \\
\hline Coil Thickness (mm) & 46.165 & 30.925 & 60.905 & 22.125 & 67.783 \\
\hline Coil Length (mm) & 201.268 & 199.492 & 110.642 & 1314.30 & 110.642 \\
\hline Current Center Axial Position (mm) & 124.00 & 564.00 & 964.00 & 1714.00 & 2464.00 \\
\hline Current Center Radial Position (mm) & 281.083 & 273.463 & 288.453 & 269.063 & 291.891 \\
\hline Coil Average $\mathrm{J}\left(\mathrm{A} \mathrm{mm}^{-2}\right)$ & 137.67 & 147.77 & 124.28 & 147.66 & 127.09 \\
\hline Number of Layers per Coil & 42 & 28 & 56 & 20 & 62 \\
\hline Number of Turns per Layer & 115 & 114 & 64 & 768 & 64 \\
\hline Total Number of Turns & 4830 & 3192 & 3584 & 15360 & 3968 \\
\hline Design Current (A) & 264.83 & 285.60 & 233.68 & 275.52 & 240.21 \\
\hline Coil Self Inductance (H) & 12.0 & 5.0 & 9.0 & 40.0 & 11.3 \\
\hline Coil Stored Energy (MJ) & 0.42 & 0.20 & 0.26 & 1.55 & 0.32 \\
\hline Peak Field in Coil $(\mathrm{T})$ & 5.30 & 4.32 & 5.68 & 4.24 & 5.86 \\
\hline Temperature Margin at $4.2 \mathrm{~K}(\mathrm{~K})$ & $\sim 1.6$ & $\sim 1.8$ & $\sim 1.5$ & $\sim 2.0$ & $\sim 1.5$ \\
\hline
\end{tabular}

\section{Measurement Apparatus and Simulations}

Axial and transverse components of the magnetic field were scanned along the axis of the solenoid warm bore tube. A stainless steel guide tube provided support for a 3D Hall probe within the warm bore (Fig. 2). The guide tube was supported on both ends by Aluminum plates which were surveyed and mounted to the warm bore openings on both sides of the magnet (Fig. 3). Measurements through the warm bore of the magnet were done along the central magnet axis $(\mathrm{x}=0 \mathrm{~mm}, \mathrm{y}$ $=0 \mathrm{~mm})$, and with $100 \mathrm{~mm}$ horizontal $(\mathrm{x}=100 \mathrm{~mm}, \mathrm{y}=0$ $\mathrm{mm})$ and vertical $(\mathrm{x}=0 \mathrm{~mm}, \mathrm{y}=100 \mathrm{~mm})$ offsets. During testing, various coil configurations were evaluated at a maximum current of 150 A ( $50 \%$ of design current). Measurements were performed at Wang NMR in Livermore (CA) in June of 2012.

Results from measurements were compared with simulations based on Opera 3D [5].

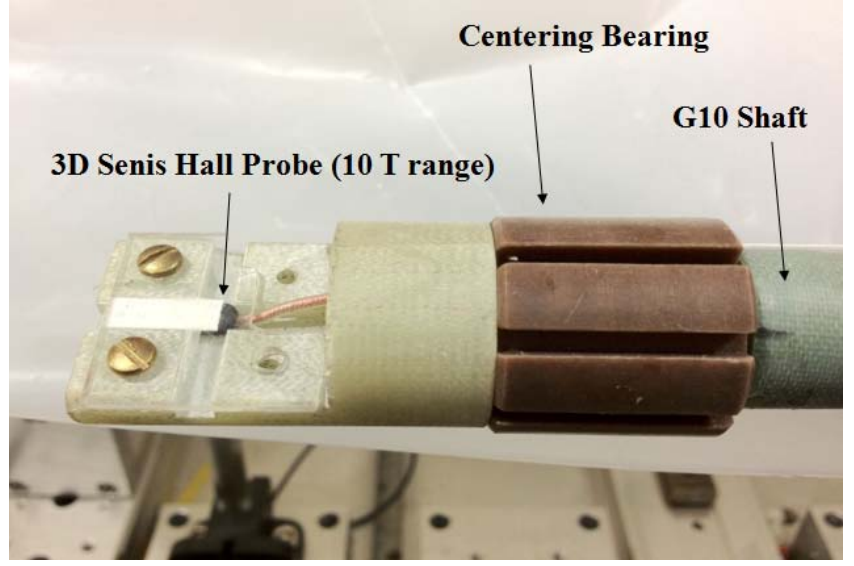

Fig. 2. 3D Hall probe attached to G10 shaft with centering bearing.

\section{SySTEMATIC UNCERTAINTIES}

The following systematic uncertainties were considered to derive a worst-case $\left(\mathrm{dB}_{\max }\right)$, and best-case, $\left(\mathrm{dB}_{\min }\right)$, uncertainty "envelop" for both axial and radial measurements along the central magnet axis (“on-axis”):

- Radial displacement of Hall probe: best-case $=2.5$ $\mathrm{mm}$, worst-case $=10 \mathrm{~mm}$ )

- Axial displacement of Hall probe (best-case $=1 \mathrm{~mm}$, worst-case $=10 \mathrm{~mm}$ )

- Tilted Hall probe (best-case $=0.1^{\circ}$, worst-case $=1^{\circ}$ )

Fig. 4 and Fig. 5 show the combined radial and axial measurement uncertainties, respectively.

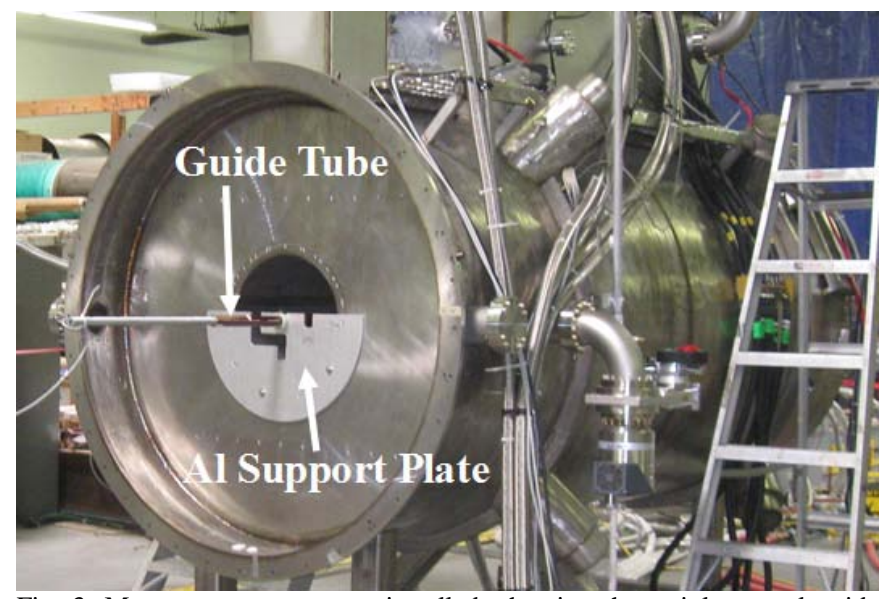

Fig. 3. Measurement apparatus installed, showing the stainless steel guide tube supported by Aluminum plates.

\section{COIL GEOMETRY VERIFICATION}

Using the as-built parameters listed in Table I, magnetic field measurements for individual coils were used to improve the geometric alignment of all coils along the magnet axis for simulations with Opera. With respect to the M1 coil, the M2, E1, C, and E2 coils appeared to be shifted towards M1 by 1.5 $\mathrm{cm}, 1.7 \mathrm{~cm}, 1.7 \mathrm{~cm}$, and $2.7 \mathrm{~cm}$, respectively. These results were used to improve the Opera model of the spectrometer solenoid for all subsequent studies.

\section{RESULTS}

Fig. 6 compares the axial component of the magnetic field $\left(B_{z}\right)$ between measurement and model for the case where all coils were powered at 150 A (on-axis). Fig. 7 shows the difference between measurement and model for the same case. 
In the spectrometer region $(\mathrm{z} \approx 100-300 \mathrm{~cm}, \mathrm{E} 1-\mathrm{C}-\mathrm{E} 2$ coils $)$ the measured magnetic field is below theoretical expectations at the level of $\sim 3 \%$. A smaller discrepancy between measurement and model can also be seen near the second matching coil $(\mathrm{z} \approx 40 \mathrm{~cm}, \mathrm{M} 2$ coil). Both features are still being investigated.

Good agreement between measurement and model for the radial components of the magnetic field is illustrated in Fig. 8, indicating small $(\sim \mathrm{mm})$ radial position offsets of the Hall probe. Off-axis measurements $(100 \mathrm{~mm}$ radial offset horizontally and vertically) of $\mathrm{B}_{\mathrm{z}}$ also display the expected behavior (Fig. 9).

\section{CONCLUSION}

Initial magnetic measurements for a MICE spectrometer solenoid were performed. Results from measurements were compared with Opera 3D simulations. Measurement uncertainties were estimated assuming “ruler-level” accuracy of the measurement. Based on measurements, coil positions along the magnet axis were adjusted to improve magnet simulations. Results from magnetic measurements were compared with simulations. Discrepancies between measurements and simulations for on-axis $B_{z}$ components of the magnetic field are under investigation.

\section{ACKNOWLEDGMENT}

The authors thank Steve Virostek, LBNL technicians, and Wang NMR personnel for their support during testing.

\section{REFERENCES}

[1] Steve Geer, "Muon Colliders and Neutrino Factories", Аnnu. Rev. of Nucl. Par. Sci. 2009.59:347-65

[2] S. P. Virostek and M. A. Green, "The Results of the MICE Spectrometer Solenoids", MICE Note $\mathbf{2 7 9}$

[3] S. P. Virostek et al., "Final Assembly and Testing of the MICE Superconducting Spectrometer Solenoids”, MICE Note $\mathbf{3 8 0}$

[4] R. Pilipenko, "An FPGA-Based Quench Detection and Continuous Logging System for Testing Superconducting Magnets”, ASC 2012, 1LPX-04

[5] Opera 3D (Vector Fields), http://www.cobham.com/aboutcobham/aerospace-and-security/about-us/antenna-systems/kidlington/ products/opera-3d.aspx

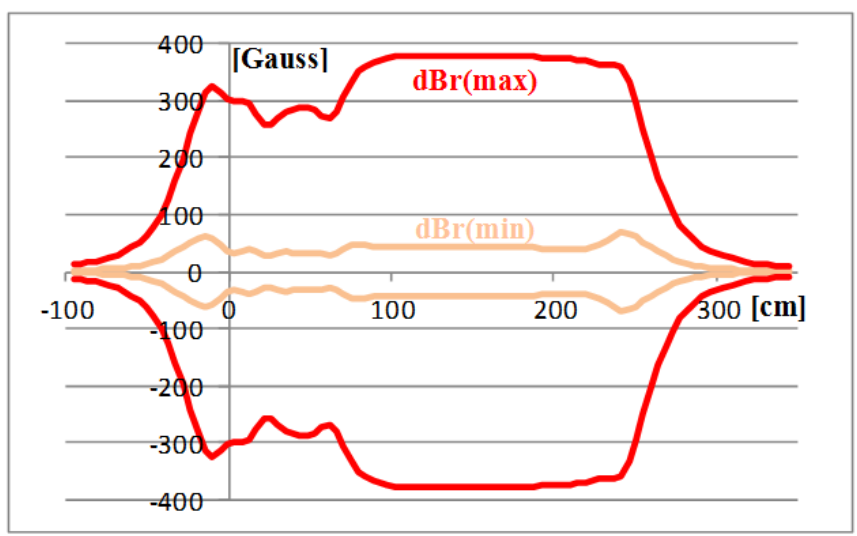

Fig. 4. Total radial measurement uncertainty $\left(\mathrm{dB}_{\mathrm{r}}, 150 \mathrm{~A}\right.$, on-axis).

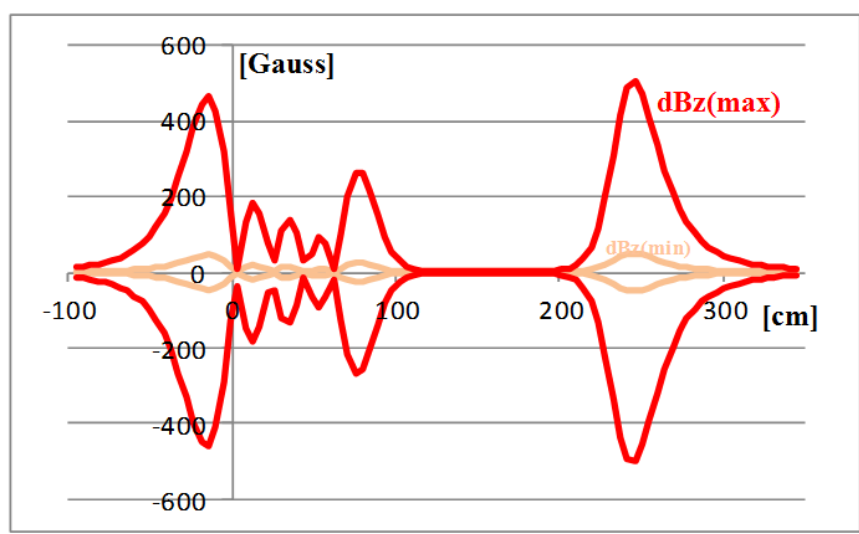

Fig. 5. Total axial measurement uncertainty $\left(\mathrm{dB}_{\mathrm{z}}, 150 \mathrm{~A}\right.$, on-axis).

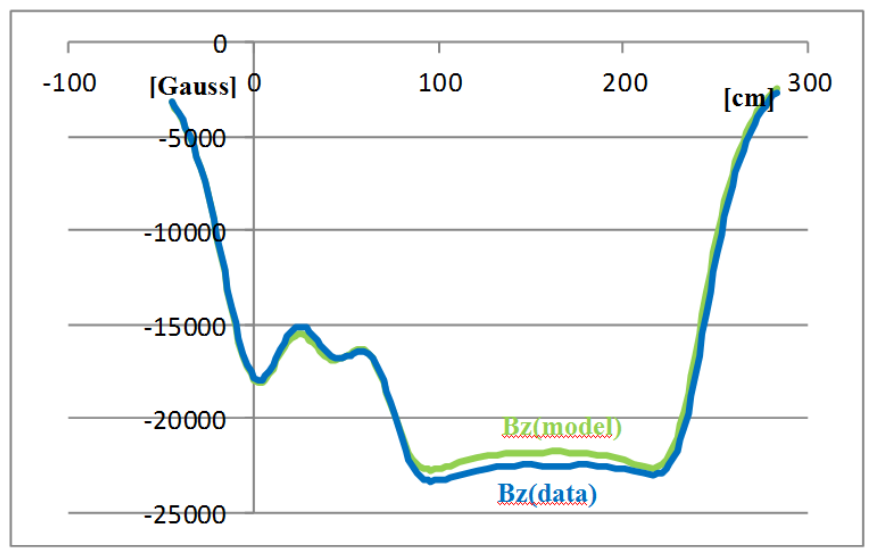

Fig. 6. Measurement vs. model for axial component of magnetic field $\left(B_{z}\right.$, $150 \mathrm{~A}$, on-axis).

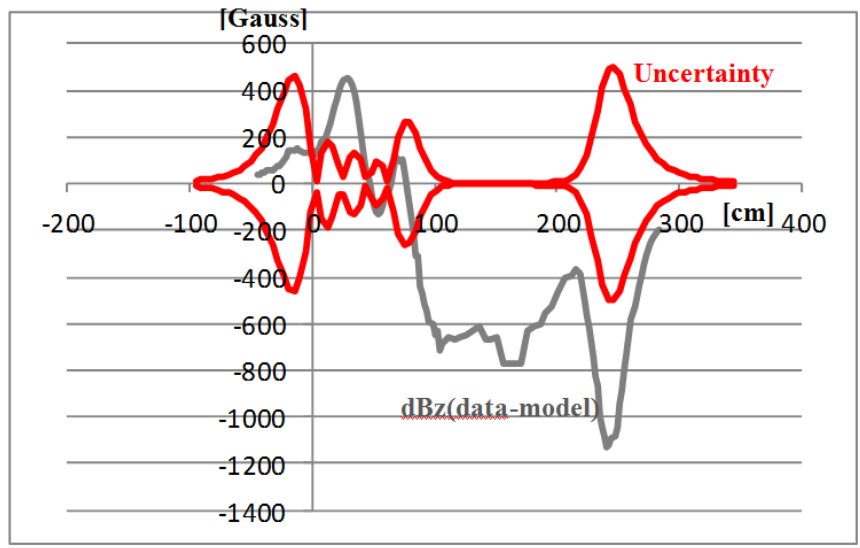

Fig. 7. Difference between measurement and model for axial component of magnetic field $\left(\mathrm{B}_{\mathrm{z}}, 150 \mathrm{~A}\right.$, on-axis). 


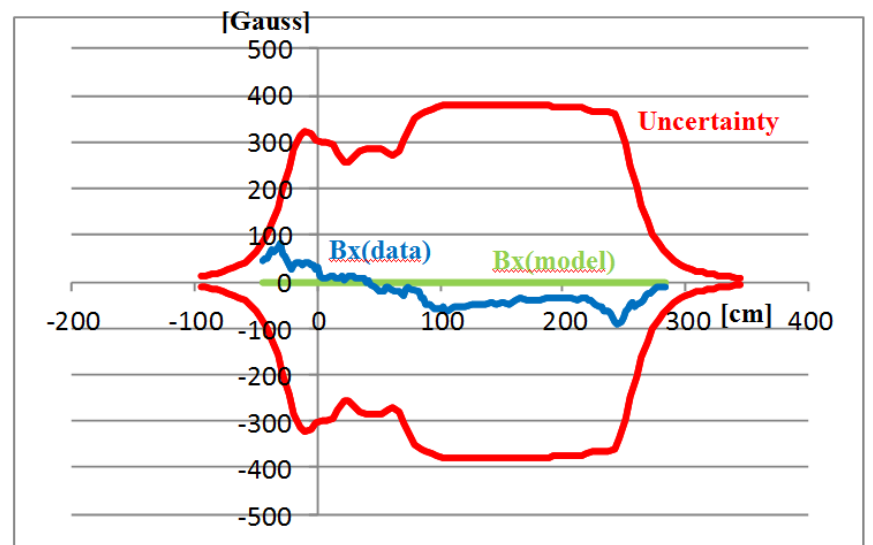

Fig. 8. Measurement vs. model for radial component of magnetic field $\left(\mathrm{B}_{\mathrm{x}}\right.$, $150 \mathrm{~A}$, on-axis).

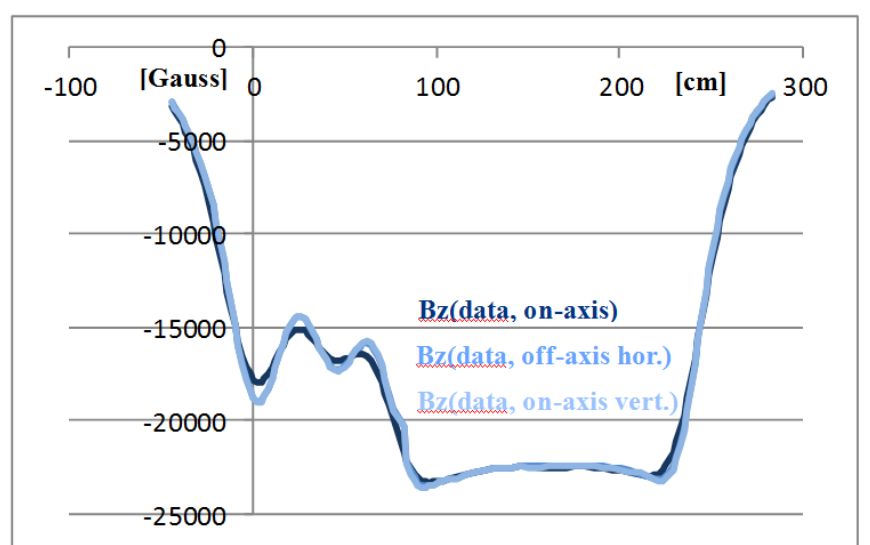

Fig. 9. On-axis vs. off-axis measurements for axial components of magnetic field (Bz, $150 \mathrm{~A}$, on-axis vs. $10 \mathrm{~cm}$ off-axis horizontally vs. $10 \mathrm{~cm}$ off-axis. 